INPLASY PROTOCOL

To cite: Song et al.

Clinicopathological and Endoscopic Features of Sessile Serrated lesions with dysplasia: Systematic review and meta-analysis. Inplasy protocol 2020110061. doi: 10.37766/inplasy2020.11.0061

Received: 15 November 2020

Published: 16 November 2020

Corresponding author: Yaoru Song

syr19960605@163.com

Author Affiliation:

The First Affiliated Hospital of Dalian Medical University

Support: NSFC:81603424.

Review Stage at time of this submission: Preliminary searches.

Conflicts of interest: We declare that we do not have any commercial or associative interest that represents a conflict of interest in connection with the work submitted.

\section{Clinicopathological and Endoscopic Features of Sessile Serrated lesions with dysplasia: Systematic review and meta-analysis}

Song, $Y^{1}$.

Review question / Objective: Sessile serrated lesions(SSL), which are precursor lesions of colorectal cancer (CRC) with BRAF mutation and the CpG island methylator phenotype (CIMP), account for up to $30 \%$ of CRC. The development of dysplasia (D) within an SSL is a critical event and represents a rapidly accelerated phase in the progression to cancer. In this study, we aimed to clarify the Clinicopathological and endoscopic features of sessile serrated lesions with dysplasia.

Condition being studied: Sessile serrated lesions(SSL), which are precursor lesions of colorectal cancer (CRC) with BRAF mutation and the CpG island methylator phenotype (CIMP), account for up to $30 \%$ of CRC. The development of dysplasia (D) within an SSL is a critical event and represents a rapidly accelerated phase in the progression to cancer.

INPLASY registration number: This protocol was registered with the International Platform of Registered Systematic Review and Meta-Analysis Protocols (INPLASY) on 16 November 2020 and was last updated on 16 November 2020 (registration number INPLASY2020110061).

\section{INTRODUCTION}

Review question / Objective: Sessile serrated lesions(SSL), which are precursor lesions of colorectal cancer (CRC) with
BRAF mutation and the CpG island methylator phenotype (CIMP), account for up to $30 \%$ of CRC. The development of dysplasia (D) within an SSL is a critical event and represents a rapidly accelerated 
phase in the progression to cancer. In this study, we aimed to clarify the Clinicopathological and endoscopic features of sessile serrated lesions with dysplasia.

Rationale: Clinicopathological and endoscopic factors will be evaluated in the meta-analysis if they were assessed factors associated with sessile serrated lesions with dysplasia. For each Clinicopathological and endoscopic factor, odds ratio (OR) was calculated for each study and as pooled estimate, with $95 \%$ confidence intervals (Cl); a P-value $<0.05$ was considered significant. Heterogeneity among studies will be assessed by using the inconsistency index (I 2 ): heterogeneity was categorised as insignificant ( $2<50 \%)$ or significant (I $2 \geq 50 \%$ ). In case of significant heterogeneity, a random effect model was used; otherwise, a fixed effect model was preferred. If possible, a subgroup analysis will be performed.

Condition being studied: Sessile serrated lesions(SSL), which are precursor lesions of colorectal cancer (CRC) with BRAF mutation and the $\mathrm{CpG}$ island methylator phenotype (CIMP), account for up to $30 \%$ of CRC. The development of dysplasia (D) within an SSL is a critical event and represents a rapidly accelerated phase in the progression to cancer.

\section{METHODS}

Search strategy: (((hyperplastic polyposis OR serrat*) AND (polyp* OR adeno* OR neoplas* OR lesion* OR precursor*) AND (colorectal OR colorectum OR colon* OR rectal OR rectum) ) AND (english[Language]) AND (humans[Filter])) NOT (Comment[Filter] OR Congress[Filter] OR Editorial[Filter] OR Letter[Filter] OR News[Filter]).

Participant or population: Patients with sessile serrated lesions with dysplasia.

Intervention: This is an observational study, we will not include any Interventions.
Comparator: Clinicopathological and endoscopic features of sessile serrated lesions without dysplasia.

Study designs to be included: All studies assessing the association of Clinicopathological and endoscopic features of sessile serrated lesions with cytological dysplasia will be included.

Eligibility criteria: All studies assessing the association of Clinicopathological and endoscopic features of sessile serrated lesions with cytological dysplasia will be included.

Information sources: Electronic databases: Cochrane, Embase, PubMed and Web of Science.

Main outcome(s): The primary outcome is the presence of cytological dysplasia.

Quality assessment / Risk of bias analysis: The risk of bias within included studies was assessed based on the QUADAS-2. Four domains related to the risk of bias were evaluated for each study, and authors' judgements were categorised as 'low', 'unclear' or 'high' risk of bias. The four domains were: (i) patient selection (i.e. if specimens were collected consecutively and without inappropriate exclusions); (ii) index test (i.e. if Clinicopathological and endoscopic features were assessed correctly and histological slides were reviewed by experienced gastrointestinal pathologists); (iii) reference standard (i.e. if there was no confusion between sessile serrated lesions with dysplasia and other serrated lesions with dysplasia ); and (iv) flow (i.e. if all included specimens underwent molecular analysis).

Strategy of data synthesis: Clinicopathological and endoscopic factors will be evaluated in the meta-analysis if they were assessed least in three studies. For each Clinicopathological and endoscopic factor, odds ratio (OR) was calculated for each study and as pooled estimate, with $95 \%$ confidence intervals (CI); a P-value <0.05 was considered significant. Heterogeneity among studies 
will be assessed by using the inconsistency index (I 2 ): heterogeneity was categorised as insignificant (I $2<50 \%$ ) or significant (I 2 $\geq 50 \%$ ). In case of significant heterogeneity, a random effect model was used; otherwise, a fixed effect model was preferred. If possible, a subgroup analysis will be performed.

Subgroup analysis: The study is in the stages of preliminary searches. Subgroup analysis will be decided whether to be performed after the extraction of data.

Sensibility analysis: The study is in the stages of preliminary searches. Sensibility analysis will be decided whether to be performed after the extraction of data.

Language: English.

Country(ies) involved: China.

Keywords: clinicopathological; colorectal polyp; serrated adenoma; colon cancer.

Contributions of each author:

Author 1 - Yaoru Song - The first author preliminarily completed the design of the study and search strategy.

More authors will join the study as it progresses. 